\title{
Clase media y democracia en América Latina
}

\author{
Jaime Fierro*
}

Perfiles Latinoamericanos | Artículo

vol. 23 | núm. 46 | 2015

pp. $37-60$

\begin{abstract}
Resumen
La consolidación de la clase media ha sido interpretada por las teorías de la modernización y de la posmodernización como un factor clave para el funcionamiento y la estabilidad del sistema democrático. Sin embargo, en América Latina la clase media ha sido asociada con dos posturas contradictorias. Por una parte, se ha destacado su papel estabilizador y democrático mientras que, por otra, ha sido vinculada con el respaldo a los golpes militares. Con la finalidad de dilucidar tal disyuntiva, se examina la relación que se puede establecer entre el nivel socioeconómico y el grado de apoyo a la democracia. Para ello, se realiza un análisis empírico a partir de las bases de datos de las encuestas Latinobarómetro, cubriendo diecisiete países de la región para el periodo 1996-2011. Se concluirá que la clase media en América Latina no posee actitudes especialmente más favorables hacia la democracia que los otros segmentos sociales.
\end{abstract}

\begin{abstract}
The consolidation of the middle class has been interpreted by modernization and postmodernization theories as a key factor for the functioning and stability of the democratic system. However, in Latin America the middle class has tended to be associated with two contradictory positions. On the one hand, it is emphasized that it plays a stabilizing and democratic role while, on the other hand, it is linked to supporting military coups. With the purpose of elucidate such a dilemma, the relationship that can be established between the socioeconomic status and the degree of support for democracy will be examined. In order to do this, an empirical analysis from Latinbarometer surveys databases will be conducted, covering seventeen countries in the region for the period from 1996 to 2011. It will be concluded that the middle class in Latin America does not have particularly more favorable attitudes toward democracy than other social segments.
\end{abstract}

Palabras clave: clase media, democracia, modernización, posmodernización, América Latina, Latinobarómetro.

Keywords: middle class, democracy, modernization, post-modernization, Latin America, Latinbarometer.

* Doctor en Ciencia Política por la Universidad de Essex, Inglaterra; doctor en Filosofía, Mención Filosofía Moral y Política por la Universidad de Chile; magíster en Ciencias Sociales y Sociólogo por la Universidad de Chile. Académico de tiempo completo en el Instituto de Asuntos Públicos (INAP) de la Universidad de Chile. 


\section{Introducción}

$\mathrm{R}$

ecientes estudios, tanto del Banco Mundial como de la CEPAL, señalan que en la última década la clase media ha aumentado en América Latina a raíz del crecimiento económico y las políticas redistributivas. Esto ha generado un fenómeno de movilidad social ascendente, integrado en su mayor parte por quienes han dejado de ser pobres (Ferreira et al., 2013; Franco et al., 2011; Hopenhayn, 2010a; León et al., 2010). No obstante, se trata de grupos socioeconómicamente vulnerables que, con una inserción laboral precaria e inestable, ingresos bajos y, en algunos casos, sin contrato ni cobertura de seguridad social, viven una situación muy frágil. Muchos de los hogares que salieron de la pobreza podrían caer nuevamente en ella (oCDE, 2010; Minujin, 2010; Mora, y Araujo, 2010; Ravallion, 2010). Por otra parte, la clase media tradicional también se ha vuelto más vulnerable en la medida en que no ha constituido una prioridad para las políticas públicas que promueve el Estado (Paramio, 2010b; Hopenhayn, 2010b).

En este contexto, algunos autores sostienen que el hecho de que los estratos medios latinoamericanos cuenten con empleos estables e ingresos razonables contribuye favorablemente a la estabilidad política nacional. En caso contrario, señalan, sus tendencias políticas pueden virar hacia opciones populistas, ya sea de izquierda o de derecha (OCDE, 2010: 15, 60). De ahí que la consolidación de la clase media sea considerada un factor clave para el funcionamiento y la estabilidad del sistema democrático (OCDE, 2010: 158).

Sin embargo, el rol democrático atribuido a la clase media no es algo que se pueda dar por sentado, a menos que la comparación con los otros segmentos sociales demuestre que efectivamente es así. Esto resulta de primera importancia, ya que "el comportamiento político de las clases medias no ha sido objeto de un estudio sistemático en años recientes" (Paramio, 2010b: 8), por lo que todavía es muy poco lo que sabemos empíricamente al respecto (López-Calva et al., 2011: 2-3), sobre todo en América Latina. Y si bien algunos han destacado el papel estabilizador y democrático de la clase media, otros la han vinculado con el respaldo a los golpes militares. Con la finalidad de dilucidar tal disyuntiva, a continuación se examina la relación que se puede establecer entre el nivel socioeconómico (NSE) y el grado de apoyo a la democracia, a partir del análisis de las bases de datos de las encuestas Latinobarómetro en diecisiete países de la región para el periodo 1996-2011. 


\section{Clase media y democracia}

Teorías de la modernización y de la posmodernización

Las teorías de la modernización de las décadas de los cincuenta y los sesenta tenían como objetivo determinar la forma en que los países subdesarrollados podrían llegar a ser sociedades desarrolladas, teniendo como referentes a Europa y Estados Unidos. Tales teorías, asociadas a autores como Seymour Martin Lipset, entre otros, vincularon el desarrollo económico con la democracia (Sztompka, 1995; Martinelli, 2005). Lipset parte del supuesto de que mientras más económicamente desarrollado sea un país, mayor será la probabilidad de que viva en democracia (Lipset, 1959a: 86; Lipset, 1960: 49-50). Incluso, a su juicio, en la medida en que aumente la cantidad de países desarrollados, la democracia se irá extendiendo alrededor del mundo (Lipset, 1959a: 75; Lipset, y Lakin, 2004: 140).

Lipset desconfiaba de los estratos más bajos (clase trabajadora) a causa del apoyo que le habían brindado al comunismo en Italia, Alemania y España, y a que se habían alineado con el populismo autoritario de Perón y Getúlio Vargas en América Latina. A su vez, consideraba que la clase trabajadora se encontraba muy influenciada por anarcosindicalistas que rechazaban la democracia burguesa en varios países de Europa y América Latina (Lipset, y Lakin, 2004: 156). De acuerdo con su hipótesis, los estratos más bajos, manteniendo el resto de las variables constantes, tienen mayores probabilidades, en relación con los otros estratos, de preferir movimientos extremistas que promueven una fácil y rápida solución a los problemas sociales. Por tanto, según Lipset, en cualquier país, la clase más baja tiende a ser más autoritaria que las clases superiores (Lipset, 1959b: 483-484).

Lipset desplazó su atención hacia la clase media, la que consideraba una fuerza estabilizadora de la democracia (Lipset, y Lakin, 2004: 12, 164). De este modo, actualiza el argumento aristotélico de que la democracia requiere bajos niveles de pobreza, porque solo así la población puede participar inteligentemente en política y no sucumbir ante los requerimientos de demagogos irresponsables. Asimismo, pensaba que la clase media podía mitigar los conflictos con grupos extremistas (Lipset, 1959a: 75, 83) y que contaba con los recursos económicos y las habilidades políticas necesarias para actuar de manera independiente frente al poder del Estado, debilitando las alianzas aristocráticas que pudieran producirse en su interior (Lipset, y Lakin, 2004: 164). Mientras que una amplia clase media facilita la existencia de la democracia, la oligarquía y la tiranía surgen con mayor probabilidad en comunidades políticas pobres y fuertemente estratificadas (Lipset, 1959a: 75; Lipset, y Lakin, 2004: 12). 
La idea de que la democracia depende en buena medida de la clase media fue duramente criticada en América Latina a finales de la década del sesenta y principios de los setenta, no solo teóricamente, sino también sobre la base de evidencia empírica. Desde el punto de vista teórico, se cuestionó que dicha perspectiva no consideraba apropiadamente los aspectos exógenos del subdesarrollo, como los efectos de la dominación colonial, el flujo del capital financiero global, y la dependencia y explotación de los países subdesarrollados por parte de los desarrollados. Los datos empíricos, por su parte, permitieron constatar que, pese a los esfuerzos de modernización de muchos países, estos no lograban alcanzar mayores niveles de desarrollo. La pobreza persistía e incluso aumentaba. Y, a su vez, el vínculo entre crecimiento económico y democracia no parecía corresponderse con lo que estaba sucediendo: la emergencia de gobiernos no democráticos o corruptos era algo frecuente, incluso entre los países que habían alcanzado un mayor nivel de desarrollo (Sztompka, 1995; Martinelli, 2005; Lipset, y Lakin, 2004; Inglehart, y Welzel, 2006).

Las posteriores teorías de la modernización, fuertemente influenciadas por la teoría de la dependencia (Furtado, 1964; Cardoso, y Faletto, 1969), tendieron a privilegiar una estrategia de desarrollo basada en la política de sustitución de importaciones. Sin embargo, dicha estrategia no logró alcanzar los efectos esperados, a diferencia de los países que optaron por orientarse hacia las exportaciones, los que en promedio experimentaron mayores niveles de crecimiento económico sostenido (Inglehart, y Welzel, 2006: 25). Hacia finales de la década de 1970, las teorías de la modernización habían perdido casi todo su atractivo y caído en abierto desuso. Este vacío fue cubierto en parte por la emergencia de las teorías de la posmodernización, que constituyeron un esfuerzo de reelaboración de sus predecesoras.

De acuerdo con Ronald Inglehart y Christian Welzel, los países posindustrializados hicieron un giro desde valores materialistas hacia valores posmaterialistas (Inglehart, 1977 y 2000; Inglehart, y Welzel, 2006; Welzel, e Inglehart, 2007 y 2008), es decir, dejaron de otorgarle una importancia fundamental a la seguridad y el crecimiento económico y empezaron a adjudicársela a la calidad de vida (Inglehart, 2000: 427-428). El crecimiento económico alcanzado habría generado las condiciones para que surgieran ciertas pautas culturales - los valores de autoexpresión - favorables a la democracia (Inglehart, 2000: 434; Welzel, e Inglehart, 2007: 307).

Los valores posmaterialistas, tales como la libertad, la diversidad y la autonomía, habrían propiciado una mayor conciencia de los derechos legales que, basados en las libertades civiles y políticas, son fundamentales para el buen funcionamiento de la democracia (Inglehart, y Welzel, 2006: 203, 209). En términos simples, quienes defienden esta perspectiva sostienen que el desarro- 
llo socioeconómico conduce a la democracia porque propicia la creación de los valores de autoexpresión, los que, a su vez, incrementan la probabilidad de que una sociedad llegue a ser democrática, continúe siéndolo o se consolide (Inglehart, y Welzel, 2006: 209-210, 227, 230). De este modo, "los valores de la autoexpresión hacen de la democracia el resultado más probable del desarrollo político" (Inglehart, y Welzel, 2006: 29), mientras que, en las sociedades que ya son democráticas, posibilitan "una forma de democracia más directa, orientada hacia cuestiones concretas y desafiante respecto a la élite", que puede transformarse "en una forma de democracia centrada en el pueblo" (Inglehart, y Welzel, 2006: 62). Aquí la clase media desempeńa un papel central: "una vez que la clase media alcanza un tamaño adecuado, su presión a favor de la democracia liberal ya no admite oposición" (Inglehart, y Welzel, 2006: 25).

Sin embargo, el rol democratizador de la clase media ya había sido puesto en duda a finales de los años sesenta y principios de los setenta en América Latina. Autores como Medina Echevarría, Faletto y Ratinnoff, entre otros, cuestionaron las virtudes atribuidas a la clase media en relación con la democracia (Hopenhayn, 2010a: 26), pues, en su opinión, la actitud que esta adopta frente a la democracia es más bien ambigua y depende del contexto económico y político de cada país.

\section{El rol de la clase media en América Latina}

Como se señaló, en América Latina la clase media ha sido asociada con dos posturas contradictorias. Por una parte, se ha destacado su papel estabilizador y democrático y, por otra, se la ha vinculado con el respaldo a los golpes militares (Paramio, 2010b: 11). En virtud de esto, la clase media es considerada "el fundamento de la estabilidad política o, por el contrario, la causa más importante de los quiebres institucionales" (Franco, y León, 2010: 62; véase también Tedesco, y Barton, 2004: 54-55). La principal debilidad de la clase media estribaría, de acuerdo con quienes sostienen este punto de vista, en que, producto de su falta de posiciones ideológicas bien definidas y estables en el tiempo, se adaptaría demasiado rápido a las coyunturas políticas, afirmación que, sin duda, cuestiona el verdadero alcance de su vocación democrática (Franco, y Hopenhayn, 2010: 15, 37; Hopenhayn, 2010a: 26; Hopenhayn, 2010b: 14-15).

La idea de que la clase media cumple un rol estabilizador y democrático se basa en la creencia de que ella posee una independencia socioeconómica relativa respecto de la clase alta, por lo que se inclinaría a frenar los proyectos expansivos de esta última si ponen en peligro sus propios intereses - intereses que 
serían antioligárquicos- (Paramio, 2010b: 11). A su vez, una clase media con mayores niveles de educación e información que las clases populares, favorecería la moderación política, particularmente cuando emergen los conflictos entre pobres y ricos, contribuyendo así a la estabilidad y reducción de la polarización (Minujin, 2010: 77; Tavares de Almeida, y Nunes de Oliveira, 2010: 104-105; Mora, y Araujo, 2010: 147; López-Calva et al., 2011: 2). De ahí que varios autores consideren que una alta proporción de clase media es una condición social necesaria para la existencia de la democracia (Tavares de Almeida, y Nunes de Oliveira, 2010: 105; Mora, y Araujo, 2010: 147; Solimano, 2010: 48).

No obstante, también hay quienes sostienen que si la clase baja pone en peligro los intereses de la clase media, esta podría aliarse con la clase alta con el fin de frenar el conflicto social o la insurrección popular. Los golpes militares en América Latina habrían sido el resultado de tales alianzas (Paramio, 2010a: 64-65; Paramio, 2010b: 11; Tedesco, y Barton, 2004: 55), como en el caso de Brasil (1964), Perú (1968), Argentina (1966 y 1976), Ecuador (1972), Chile (1973) y Uruguay (1973) (Smith, 2005: 59).

Dicha explicación, sin embargo, ha sido matizada por algunos autores que sostienen que esta "confunde la amenaza de una revolución social con una situación prolongada de desorden social y político" (Paramio, 2010b: 11). En otras palabras, si bien los golpes militares fueron justificados como una respuesta frente a la amenaza revolucionaria, el grado de apoyo que tuvieron en la clase media se debió principalmente a la situación de desorden social y político que experimentaban los países en que se produjeron, algo que sería muy claro en el caso de Brasil en 1964, donde no existía ninguna amenaza revolucionaria real (Paramio, 2010b: 11).

De lo anterior se desprende que las orientaciones de la clase media hacia la democracia operan de manera "contingente" —y no incondicional- en función de ciertos contextos sociopolíticos y socioeconómicos relevantes. En particular, la clase media no necesariamente apoyaría los procesos de democratización cuando siente temor a que la inestabilidad política afecte sus privilegios (Chen, 2013: 5-6). Y puesto que los intereses de la clase media, más allá de promover su propia inclusión, no están siempre del todo claros, su posición intermedia en la estructura de clases la hace potencialmente atractiva para los elementos antidemocráticos que pretenden formar alianzas dentro de la clase dominante, que es la dueña y la que controla el capital (Doorenspleet, 2002: 50). En América Latina, durante el periodo 1940-1977, la clase media habría llevado a cabo una política de alianzas oportunista, especialmente en lo que respecta a las élites o los militares (Smith, 2005: 58-59): en algunos casos apoyó el llamado democrático por el cambio social, mientras que en otros lo resistió (Smith, 2005: 321). 
De acuerdo con esta perspectiva, es un error caracterizar las actitudes de la clase media como inherentemente más democráticas que las del resto de los segmentos sociales. Por el contrario, es altamente probable que la relación entre clase media y democracia sea mucho más débil de lo que algunas teorías — particularmente las de la modernización y las de la posmodernización— han tendido a pensar. Trabajos recientes pondrían de manifiesto que, en el mundo, la clase media se inclina a dar la espalda a la democracia en contextos de caos, corrupción, débil crecimiento económico o temor, frente a la disminución de su propio poder (Kurlantzick, 2013: 32). En otras palabras, para algunos, "el mito de la clase media como actor social y fuerza política tiende a difuminarse" (Franco, y Hopenhayn, 2010: 8).

Por otra parte, en la teorización acerca del apoyo a la democracia se suele considerar la dimensión de la adhesión a los principios del régimen democrático (Norris, 1999), en términos de que la democracia es preferible a cualquier otra forma de gobierno, algo que es fundamental para hacerla viable y mantenerla en el tiempo (Easton, 1975; Huntington, 1993; Diamond, 1999; Klingemann, 1999; Dalh, 2000; Dalton, 1999 y 2004; Inglehart, y Welzel, 2006; Welzel, e Inglehart, 2007; Booth, y Seligson, 2009; Norris, 1999 y 2011). Y si bien los estudios de opinión pública no suelen clarificar qué significa el término "democracia" para los encuestados, algunas investigaciones han puesto de manifiesto que las definiciones que estos manejan se ajustan razonablemente al significado de la misma (Sarsfield, 2010: 30-31). Así, por ejemplo, frente a la pregunta abierta “¿Qué significa democracia?", que formuló Latinobarómetro en 2001, 2002, 2005 y 2006, los latinoamericanos respondieron en promedio: "libertades civiles e individuales" (39\%), "igualdad y justicia" (12\%) y "derecho a voto" (7\%) (Latinobarómetro, 2006: 54). Por lo tanto, en América Latina (así como en Asia y África), los ciudadanos no poseen un concepto de democracia vago o vacío de contenido, como tampoco uno asociado principalmente a las necesidades económicas, sino uno vinculado con las libertades civiles e individuales (Dalton, Shin, y Jou, 2008: 1-2, 5-6). Atendiendo a tales consideraciones, a continuación se precisan los aspectos metodológicos para estudiar la relación entre el NSE y el apoyo a la democracia en América Latina.

\section{Aspectos metodológicos}

\section{Universo y muestra}

Para la realización del análisis empírico se utilizaron las bases de datos disponibles de las encuestas Latinobarómetro para el periodo 1996-2011, en las que 
se incluyen mediciones sistemáticas de diecisiete países latinoamericanos. Las encuestas son estadísticamente representativas del 100\% de la población adulta de cada país, con un margen de error estimado en torno al $\pm 3 \%$. En la medición del año 2011, se aplicó un total de 20204 entrevistas. Por otra parte, gracias a que las encuestas Latinobarómetro utilizan instrumentos estandarizados, es posible comparar los países a través del tiempo.

Variable dependiente: apoyo a la democracia

La variable "apoyo a la democracia" intenta captar las orientaciones normativas de los ciudadanos hacia la democracia, en términos de la deseabilidad de un régimen democrático (Sarsfield, 2010: 28-31; Norris, 1999 y 2011). La pregunta (P13ST) se formula en el cuestionario del siguiente modo: “¿Con cuál de las siguientes frases está Ud. más de acuerdo? 1 = la democracia es preferible a cualquier forma de gobierno; 2 = en algunas circunstancias, un gobierno autoritario puede ser preferible a uno democrático; 3 = a la gente como uno, nos da lo mismo un régimen democrático que uno no democrático". Esta pregunta se utiliza para el análisis bivariado de la sección "Apoyo a la democracia según NSE en América Latina”.

Con la finalidad de evaluar mejor el impacto de la variable NSE sobre la adhesión a la democracia, se ha construido un Índice de Apoyo a la Democracia para el año $2011 .{ }^{1}$ Dicho índice ha sido elaborado a partir de tres preguntas independientes entre sí (P13ST, P16ST.A y P18ST), seleccionándose un indicador en cada una de ellas: $i$ ) la democracia es preferible a cualquier otra forma de gobierno; ii) la democracia puede tener problemas, pero es la mejor forma de gobierno; iii) en ninguna circunstancia apoyaría un gobierno militar. Cada uno de los indicadores fue recodificado en valores 0 y 1 (variables dummy), ${ }^{2}$ por lo que el índice final sumatorio asume cuatro valores, que fluctúan entre 0 y 3. El Índice de Apoyo a la Democracia es incluido como variable dependiente en el análisis multivariado de la sección "Determinantes del apoyo a la democracia: la incidencia del NSE".

1 Esto reviste cierta relevancia puesto que, en lo que respecta al grado de legitimidad de la democracia, "existe un consenso creciente sobre la necesidad de utilizar más de un indicador para medirla e idealmente sobre la importancia de construcción de índices" (Sarsfield, 2010: 26). Y, a su vez, permite corregir en parte el sesgo de "deseabilidad social" asociado a la formulación de este tipo de preguntas (aumentando la validez del instrumento).

2 Los NS y NR fueron imputados como valor "0", ya que interesa resaltar la presencia del atributo en cuestión. 
Son varios los estudios de opinión pública sobre la clase media en los que se utilizan medidas subjetivas basadas en la autopercepción, ya sea del NSE o bien sobre la posición en la distribución del ingreso. ${ }^{3}$ Sin embargo, se ha demostrado que existen importantes diferencias entre sus resultados y los que arrojan los estudios que usan medidas más objetivas vinculadas, por ejemplo, al ingreso efectivo (López-Calva, 2011: 3; Ferreira et al., 2013: 166). En las encuestas Latinobarómetro se utiliza "la apreciación del NSE" del entrevistado por parte del encuestador — como un proxy de ingresos y, por ende, de clase social— a partir de tres indicadores: calidad de la vivienda, calidad del amueblado, y apariencia general del entrevistado. Cada individuo es clasificado en alguna de las cinco categorías: muy buena, buena, regular, mala, o muy mala. ${ }^{4}$ De este modo, se trabaja con cuatro categorías de NSE: 1 = bajo (muy mala + mala); $2=$ medio bajo (regular); 3 = medio alto (buena), y 4 = alto (muy buena). Al separarla en dos subgrupos, medio bajo y medio alto, este procedimiento permite registrar la heterogeneidad de la clase media.

\section{Variables de control}

Las "características sociodemográficas" suelen ser incorporadas como variables de control al momento de estudiar las determinantes de las actitudes hacia la democracia (Sarsfield, 2010: 35-36; Booth, y Seligson, 2009: 106-107). Esto no solo porque desempeñan un papel relevante en la configuración del proceso de socialización política, sino también porque podrían afectar las actitudes de la clase media hacia la democracia. Aquí se considerarán las variables sexo, edad y escolaridad. 5 Adicionalmente, se incluye la variable autopercepción de

3 Este sería el caso, por ejemplo, del estudio de la OCDE (2010: 162-164), Perspectivas económicas para América Latina 2011, en el que se analizan las actitudes hacia la democracia según quintiles de ingreso subjetivo (autoposicionamiento de los entrevistados).

4 Y si bien se trata de una clasificación no exenta de críticas, al compararla con estimaciones nacionales objetivas de ingresos, existe una buena correspondencia entre el agrupamiento de las categorías "muy mala" y "mala" y los niveles de pobreza de los países latinoamericanos, mientras que la categoría "muy buena" tiende a corresponder al segmento $\mathrm{ABC} 1$, comúnmente utilizado en estudios de opinión pública y estudios de mercado.

5 La recodificación de las variables es la siguiente: sexo $(0=$ hombre; $1=$ mujer $)$, edad $(1=16-25 ; 2=26$ $40 ; 3$ = 41-60; 4 = 61 y más), escolaridad ( 1 = analfabeto; 2 = básica incompleta; 3 = básica completa; 4 = secundaria, media, técnica incompleta; 5 = secundaria, media, técnica completa; $6=$ superior completa; 7 = superior incompleta). 
ingresos razonables, ${ }^{6}$ ya que, como se ha indicado, podría influenciar las actitudes políticas de la clase media (OCDE, 2010: 15, 60).

La "confianza interpersonal"7 se encuentra entre los principales valores asociados al desarrollo democrático (Elgströn, 2002: 192, 196). Diversos estudios han encontrado - tanto en las democracias desarrolladas como en las nuevasque quienes confían en los otros apoyan más la democracia (Dalton, 2004: 70; Sarsfield, 2010: 38).

De igual modo, se ha argumentado que el efecto del grado de "exposición a los medios de comunicación" 8 sobre el apoyo a la democracia puede ser tanto positivo como negativo (Dalton, 2004: 71-4; Booth, y Seligson, 2009: 116). En el primer caso, se afirma que los medios de comunicación hacen que la información política sea más accesible para la población, lo que ayudaría al desarrollo de competencias ciudadanas que favorecerían las actitudes democráticas. En el segundo, se señala que la exposición a las noticias (que tienden a trivializar la política) genera desencanto y desafección hacia la política y, por extensión, hacia el régimen político en general (Gunther et al., 2007a: 18-20; Gunther et al., 2007b: 333-334; 23-24; Schimitt-Beck, y Voltmer, 2007: 75-84).

Por otra parte, entre los factores que se han identificado comúnmente para explicar el nivel de apoyo a la democracia, se encuentran la "evaluación de la situación económica” — tanto a nivel familiar como del país— 9 y la "evaluación de la situación política” —en términos de la aprobación de la gestión del gobierno ${ }^{10}$ y la satisfacción con la situación política— ${ }^{11}$ que hacen los ciudadanos. Las actitudes hacia la democracia dependerán de las evaluaciones económicas y políticas que se hagan, en una relación directamente proporcional (Sarsfield, 2010: 10, 12, 36-37; Chu et al., 2008: 32-33, 42).

Finalmente, se ha enfatizado que la "satisfacción con el funcionamiento de la democracia" 12 se encuentra entre las variables con mayor incidencia en el apoyo a la democracia (Chu et al., 2008: 37). De acuerdo con la ocDe (2010: $157,161,178)$, los estratos medios latinoamericanos defienden el concepto de democracia, pero son críticos con el funcionamiento del sistema político.

\footnotetext{
6 Recodificación: 1 = no les alcanza, tienen grandes dificultades; 2 = no les alcanza, tienen dificultades; 3 = les alcanza justo, sin grandes dificultades; 4 = les alcanza bien, pueden ahorrar (recodificación ascendente).

7 Recodificación: 0 = uno nunca es lo suficientemente cuidadoso en el trato con los demás; 1 = se puede confiar en la mayoría de las personas.

8 Recodificación: número de días en la semana en que ve noticias políticas en televisión (TV) abierta.

9 En ambos casos se recodifica como: 1 = muy mala; 2 = mala; 3 = regular; 4 = buena; 5 = muy buena.

10 Recodificación: 0 = no; 1 = sí.

11 Recodificación: 1 = muy mala; 2 = mala; 3 = regular; 4 = buena; 5 = muy buena.

12 Recodificación: 1 = nada satisfecho; 2 = no muy satisfecho; 3 = más bien satisfecho; 4 = muy satisfecho.
} 


\section{Apoyo a la democracia según NSE en América Latina}

El apoyo a la democracia ha sido usualmente considerado un elemento determinante para la legitimidad y estabilidad de un régimen democrático, particularmente en aquellas sociedades que experimentan o han experimentado transiciones a la democracia. En virtud de esto, lo primero que llama la atención es que, luego de transcurridas más de dos décadas desde el retorno a la democracia en América Latina, los niveles de adhesión a esta sean todavía bajos. Dicha tendencia se ha mantenido durante el periodo 1996-2011. A su vez, el nivel de apoyo alcanzado en 2011 (62\%) es incluso más bajo del que existía en la segunda mitad de los noventa (tabla 1 ).

Por otra parte, es interesante constatar que la caída del PIB entre 2007 y 2009 (de $5.5 \%$ a $3.1 \%$ ) no estuvo asociada a un descenso en los niveles de apoyo a la democracia en la región; de hecho, estos aumentaron de 59\% a 66\% durante el periodo, tendencia que se puede verificar en todos los segmentos sociales. Y, a la inversa, el incremento del PIB entre 2002 y 2004 (de -2.3\% a 4.4\%) tampoco estuvo asociado a un aumento en el nivel de apoyo a la democracia, el que bajó de $63 \%$ a $60 \%$. Esto tiende a corroborar la idea de que los ciudadanos latinoamericanos distinguen cada vez más la democracia como un sistema de gobierno del desempeño del gobierno de turno, tendiendo incluso a aumentar su apoyo a la primera en momentos de crisis económicas (Graham, y Sukhtankar, 2004: 349-350, 370, 375).

Tabla 1. Apoyo a la democracia según NSE (\%) América Latina, 1996-2011

\begin{tabular}{lcccccccccccccccc}
\hline NSE & 1996 & 1997 & 1998 & 2000 & 2001 & 2002 & 2003 & 2004 & 2005 & 2006 & 2007 & 2008 & 2009 & 2010 & 2011 & Media \\
\hline Bajo & 61 & 62 & 57 & 56 & 48 & 58 & 54 & 55 & 57 & 58 & 54 & 55 & 60 & 65 & 57 & 57 \\
Medio bajo & 64 & 66 & 63 & 63 & 53 & 63 & 58 & 59 & 59 & 62 & 58 & 61 & 62 & 65 & 61 & 61 \\
Medio alto & 67 & 69 & 69 & 66 & 59 & 67 & 60 & 63 & 63 & 66 & 62 & 65 & 67 & 68 & 65 & 65 \\
Alto & 66 & 70 & 69 & 69 & 59 & 65 & 65 & 69 & 69 & 67 & 64 & 68 & 71 & 68 & 68 & 67 \\
$\begin{array}{l}\text { América } \\
\text { Latina }\end{array}$ & 64 & 66 & 65 & 63 & 55 & 63 & 58 & 60 & 61 & 63 & 59 & 62 & 64 & 66 & 62 & 62 \\
\hline
\end{tabular}

Nota: Aquí solo se muestra la categoría "la democracia es preferible a cualquier otra forma de gobierno". Se excluyen los NS/NR.

Fuente: Elaboración propia a partir de las bases de datos de las encuestas Latinobarómetro, 1996-2011.

En el ańo 2011, solo seis de los diecisiete países estudiados presentan niveles de apoyo a la democracia por sobre el 66\%: Argentina (71\%), Costa Rica (69\%), Panamá (67\%), Uruguay (79\%), Venezuela (79\%) y Bolivia (70\%) (tabla 3). Sin embargo, tales valores se reducen significativamente si se utilizan 
instrumentos más exigentes para medir la adhesión a los principios democráticos, como el Índice de Apoyo a la Democracia. De acuerdo con dicho índice, tenemos que Argentina cae al 59\%, Costa Rica al 51\%, Panamá al 42\%, Uruguay al $62 \%$, Venezuela al $53 \%$ y Bolivia al $41 \%$. Es decir, de los diecisiete países analizados, únicamente cuatro presentan niveles de apoyo a la democracia por sobre el $50 \%$ (con respuestas positivas de adhesión a la democracia en los tres indicadores del índice). ${ }^{13}$

Por otra parte, al examinar esta tendencia en el periodo considerado (19962011), es posible constatar que en América Latina, a medida que aumenta el NSE, tiende a aumentar también la proporción de quienes consideran que la democracia es la mejor forma de gobierno. En promedio, el segmento medio bajo presenta un $61 \%$ de apoyo y el medio alto un $65 \%$, en comparación con el $57 \%$ del bajo y el $67 \%$ del alto (tabla 1 ).

Sin embargo, un análisis por países nos revela que dicha tendencia es más bien débil a través del tiempo: no existe a lo largo de varios ańos y es particularmente inconsistente en países como Bolivia, Costa Rica, Ecuador, Honduras, Nicaragua, Panamá, Paraguay y Venezuela (tabla 2). Tales resultados sugieren que el apoyo a la democracia depende del contexto sociopolítico y socioeconómico de los países latinoamericanos, aunque muy probablemente el segundo sea de menor importancia (según se examinó a propósito de las fluctuaciones del рів entre los años 2002-2004 y 2007-2009)..$^{14}$ En tal sentido, como se mostrará más adelante para el año 2011, la "aprobación de la gestión del presidente" y la "satisfacción con el funcionamiento de la democracia" son las dos variables que mayor impacto tienen sobre el Índice de Apoyo a la Democracia en la mayoría de los países latinoamericanos, a diferencia de la "evaluación de la situación económica del país" o la "evaluación de la situación económica personal" (tabla 4).

13 Los valores del Índice de Apoyo a la Democracia para el resto de los países con respuestas positivas de adhesión a la democracia en los tres indicadores son los siguientes: Guatemala (8\%), México (16\%), Honduras (19\%), El Salvador (26\%), Brasil (32\%), Perú (32\%), Nicaragua (32\%), Paraguay (33\%), Colombia (35\%), Panamá (42\%), Ecuador (44\%) y Chile (50\%).

14 De manera análoga, en un estudio que se llevó a cabo en seis países latinoamericanos a partir de las bases de datos de la encuesta Ecosocial 2007 (Argentina, Brasil, Chile, Colombia, Guatemala, México y Perú), si bien se constata a nivel del total de la muestra que existe una correlación entre ingresos y un conjunto de valores políticos (como, por ejemplo, la confianza en las instituciones), al controlar por el efecto de los países, se concluye que el ingreso se encuentra débilmente asociado con dichos valores, siendo hasta cierto punto determinado por los distintos contextos. Y, por lo tanto, se sugiere que dicha evidencia socava la idea de que las clases medias posean valores (democráticos) comunes a través de América Latina (Ferreira et al., 2013: 171). 
Tabla 2. Correlación entre apoyo a la democracia y NSE por países de América Latina, 1996-2011

\begin{tabular}{|c|c|c|c|c|c|c|c|c|c|c|c|c|c|c|c|c|}
\hline País & 1996 & 1997 & 1998 & 2000 & 2001 & 2002 & 2003 & 2004 & 2005 & 2006 & 2007 & 2008 & 2009 & 2010 & 2011 & Total \\
\hline Argentina & Sí & Sí & Sí & Sí & Sí & Sí & Sí & Sí & . & Sí & Sí & Sí & Sí & Sí & Sí & 14 \\
\hline Bolivia & Sí & . & . & Sí & ${ }^{\circ}$ & . & Sí & . & Sí & & & & & Sí & . & 6 \\
\hline Brasil & Sí & & & Sí & Sí & Sí & Sí & Sí & Sí & Sí & Sí & Sí & Sí & & & 11 \\
\hline Chile & Sí & & Sí & Sí & Sí & Sí & Sí & Sí & Sí & Sí & . & Sí & . & Sí & Sí & 12 \\
\hline Colombia & Sí & . & . & Sí & Sí & Sí & Sí & Sí & Sí & . & Sí & Sí & Sí & Sí & Sí & 12 \\
\hline Costa Rica & . & . & & & Sí & ${ }^{\circ}$ & Sí & Sí & . & . & Sí & & . & Sí & & 5 \\
\hline Ecuador & . & & Sí & . & Sí & Sí & & & Sí & . & . & Sí & . & & . & 5 \\
\hline EI Salvador & & Sí & Sí & Sí & Sí & Sí & . & & & Sí & & Sí & Sí & & . & 8 \\
\hline Guatemala & & Sí & . & . & Sí & Sí & Sí & . & Sí & & Sí & Sí & & . & Sí & 8 \\
\hline Honduras & . & & Sí & . & . & & & Sí & & Sí & . & & . & Sí & & 4 \\
\hline México & Sí & Sí & Sí & Sí & & & & Sí & & Sí & Sí & Sí & Sí & Sí & Sí & 11 \\
\hline Nicaragua & . & r & ${ }^{\prime}$ & Sí & Sí & Sí & . & . & . & . & & & Sí & - & 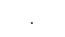 & 4 \\
\hline Panamá & . & . & & & Sí & . & . & Sí & Sí & . & & & . & Sí & & 4 \\
\hline Paraguay & Sí & & . & . & Sí & Sí & & & Sí & Sí & Sí & & . & & . & 6 \\
\hline Perú & & Sí & Sí & . & Sí & . & Sí & & & Sí & Sí & Sí & Sí & Sí & Sí & 10 \\
\hline Uruguay & & . & Sí & 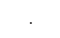 & Sí & Sí & Sí & . & & & Sí & Sí & Sí & Sí & Sí & 9 \\
\hline Venezuela & & & & . & Sí & & & & & & . & Sí & . & Sí & Sí & 4 \\
\hline Total & 7 & 5 & 8 & 8 & 14 & 10 & 9 & 8 & 8 & 8 & 9 & 11 & 8 & 11 & 8 & \\
\hline
\end{tabular}

Nota: La expresión "Sí" corresponde a una correlación (Spearman) estadísticamente significativa; p < 0,05.

Fuente: Elaboración propia a partir de las bases de datos de las encuestas Latinobarómetro, 1996-2011.

Tabla 3. Apoyo a la democracia según NSE (\%) por países de América Latina, 2011

\begin{tabular}{|c|c|c|c|c|c|c|}
\hline \multirow{2}{*}{ País } & \multirow{2}{*}{ Ingreso per cápita* } & \multicolumn{4}{|c|}{ NSE } & \multirow[b]{2}{*}{ Total } \\
\hline & & Bajo & Medio bajo & Medio alto & Alto & \\
\hline Argentina & Medio alto & 67 & 66 & 75 & 68 & 71 \\
\hline Brasil & Medio alto & 55 & 48 & 51 & 65 & 52 \\
\hline Chile & Medio alto & 54 & 65 & 65 & 61 & 63 \\
\hline Colombia & Medio alto & 45 & 55 & 59 & 80 & 59 \\
\hline Costa Rica & Medio alto & 73 & 67 & 70 & 70 & 69 \\
\hline Ecuador & Medio alto & 60 & 62 & 69 & 61 & 64 \\
\hline México & Medio alto & 38 & 43 & 51 & 65 & 44 \\
\hline Panamá & Medio alto & 56 & 67 & 69 & 66 & 67 \\
\hline Perú & Medio alto & 52 & 63 & 70 & 77 & 64 \\
\hline Uruguay & Medio alto & 70 & 77 & 82 & 83 & 79 \\
\hline Venezuela & Medio alto & 79 & 75 & 83 & 89 & 79 \\
\hline Bolivia & Medio bajo & 73 & 70 & 69 & 68 & 70 \\
\hline El Salvador & Medio bajo & 55 & 60 & 56 & 60 & 58 \\
\hline Guatemala & Medio bajo & 46 & 41 & 36 & 38 & 41 \\
\hline Honduras & Medio bajo & 43 & 48 & 48 & 43 & 46 \\
\hline Nicaragua & Medio bajo & 60 & 62 & 56 & 56 & 60 \\
\hline Paraguay & Medio bajo & 53 & 54 & 60 & 53 & 56 \\
\hline
\end{tabular}

* Banco Mundial, 2011, utilizando el método Atlas.

Nota: Aquí solo se muestra la categoría "la democracia es preferible a cualquier otra forma de gobierno". Se excluyen los NS/NR.

Fuente: Elaboración propia a partir de la base de datos de la encuesta Latinobarómetro 2011. 
Por otra parte, si se considera el año 2011, se constata que solo en ocho de los diecisiete países bajo estudio se da una relación estadísticamente significativa entre el NSE y el apoyo a la democracia, siendo dicha relación más clara en los países de ingreso per cápita medio alto, a diferencia de los de ingreso medio bajo (tabla 3). Tales resultados tienden a respaldar parcialmente tanto las aproximaciones de la teoría de la modernización como las de la teoría de la posmodernización. Sin embargo, en países como Colombia, México, Perú y Venezuela, aunque la clase media apoya más a la democracia que la clase baja, lo hace en una menor proporción que la clase alta. Y en el caso de Guatemala, por otra parte, la relación es inversa: a medida que disminuye el NSE, aumenta la proporción de quienes consideran que la democracia es preferible a cualquier otra forma de gobierno (tabla 3 ).

Del mismo modo, cabe destacar que la hipótesis propuesta por Lipset (1959b: 483-484), según la cual la clase más baja en cualquier país tiende a ser más autoritaria que las clases superiores, solamente se verifica en ocho de los diecisiete países bajo estudio en 2011 (tabla 3). Ciertamente, tales resultados no son concluyentes, razón por la que es necesario retestearlos mediante un análisis multivariado que incluya un número razonable de variables de control.

\section{Determinantes del apoyo a la democracia: la incidencia del NSE}

Aunque el presente estudio no tiene por finalidad establecer un modelo que tenga una particular capacidad explicativa respecto de los niveles de apoyo a la democracia en América Latina, sí resulta importante estimar el impacto del NSE sobre el Índice de Apoyo a la Democracia. Para evitar posibles relaciones espurias entre ambas variables, se ha introducido un conjunto de variables de control que la literatura considera relevantes, según se examinó previamente en el apartado metodológico. Los resultados del análisis multivariado (regresión lineal múltiple) se presentan por países para el año 2011, los que han sido agrupados de acuerdo con su nivel de desarrollo, según la clasificación del método Atlas del Banco Mundial. A partir de las tablas 4 y 5, es posible afirmar que:

- Características sociodemográficas: de las variables consideradas, solo la escolaridad posee un impacto estadísticamente significativo y persistente sobre el Índice de Apoyo a la Democracia, y que dicha relación es más clara en los países de ingreso medio alto (con la excepción de Venezuela) que en los de ingreso medio bajo.

- Confianza interpersonal: contrario a lo que sugieren las teorías del capital social, la confianza interpersonal posee una escasa o nula incidencia sobre el Índice de Apoyo a la Democracia, tanto en los países de ingreso 
medio alto como en los de ingreso medio bajo. A su vez, la relación es inversa a la esperada en cinco de los seis países en los que se verifica dicha relación (la excepción es Argentina). Es decir, quienes señalan que se puede confiar en la mayoría de las personas tienden a apoyar menos la democracia que quienes no lo hacen.

- Exposición a los medios (TV): el número de días a la semana en que las personas ven noticias en televisión tiene una escasa o nula incidencia sobre el Índice de Apoyo a la Democracia en ambos grupos de países, aunque con un efecto positivo. En los siete países en los que se verifica dicha relación, se observa que a medida que aumenta el número de días en que las personas ven noticias, aumenta el apoyo a la democracia.

- Evaluación de la situación económica: ni la evaluación de la situación económica personal ni la de la situación económica del país poseen una incidencia estadísticamente significativa sobre el Índice de Apoyo a la Democracia en la mayoría de los países examinados.

- Evaluación de la situación política: la aprobación de la gestión del presidente tiende a incidir significativamente en el Índice Apoyo a la Democracia, a diferencia de lo que ocurre con la calificación de la situación política del país, tendencia que se puede observar en los países de ingreso medio alto (seis de once). Por otra parte, en Ecuador, México, Panamá, Uruguay y Paraguay, quienes aprueban la gestión del presidente tienden a apoyar más la democracia que quienes la desaprueban, mientras que tanto en Chile como en Perú se da la tendencia contraria.

- Satisfacción con la democracia: la satisfacción con el funcionamiento de la democracia impacta fuertemente sobre el apoyo a la democracia, y es esta la variable de mayor importancia en el modelo en ambos grupos de países (con la excepción de Ecuador).

- La capacidad explicativa del modelo es moderada en la mayoría de los países (sobre el 10\%), lo que es particularmente relevante si se tiene en consideración que se está estudiando el apoyo a la democracia a "nivel individual”. Las variables que más contribuyen a explicar la varianza en el Índice de Apoyo a la Democracia son la escolaridad y la satisfacción con el funcionamiento de la democracia.

- Finalmente, dentro de las características sociodemográficas, al examinar el impacto del NSE sobre el Índice de Apoyo a la Democracia por países, introducidas las variables de control, se constata que el NSE posee nula incidencia en quince de los diecisiete países estudiados. Y, de los dos en que se verifica dicha relación, el caso de Colombia confirma que a medida que aumenta el NSE de las personas se incrementa el apoyo a la democracia, mientras que en el caso de Chile se da la relación inversa. 
Con ello se diluye —en el "análisis a nivel individual" — la supuesta relación entre clase media y democracia, propiciada por las teorías de la modernización y las de la posmodernización, ${ }^{15}$ por lo menos para América Latina. Y, del mismo modo, se confirma el descarte de la hipótesis de que el NSE bajo, en cualquier país, tiende a ser más autoritario que los segmentos de estratos superiores.

Tabla 4. Determinantes del Índice de Apoyo a la Democracia en países con ingreso per cápita medio alto, 2011

\begin{tabular}{|c|c|c|c|c|c|c|c|c|c|c|c|}
\hline & 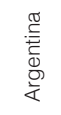 & $\begin{array}{l}\overline{\bar{g}} \\
\bar{\pi} \\
\bar{\infty}\end{array}$ & $\begin{array}{l}\frac{\pi}{0} \\
\frac{0}{E} \\
\frac{0}{0} \\
U\end{array}$ & $\begin{array}{l}\tilde{J} \\
\frac{0}{\pi} \\
\widetilde{J} \\
\tilde{D} \\
0 \\
0\end{array}$ & $\frac{\stackrel{\omega}{T}}{\frac{\pi}{U}}$ & $\begin{array}{l}\bar{o} \\
\frac{0}{0} \\
\bar{J} \\
\dot{U}\end{array}$ & $\begin{array}{l}.0 \\
.0 \\
\sum^{0}\end{array}$ & 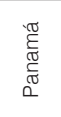 & $\frac{0}{0}$ & 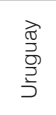 & $\begin{array}{l}\frac{\pi}{0} \\
\frac{N}{N} \\
\mathbb{Q} \\
\frac{D}{D}\end{array}$ \\
\hline \multicolumn{12}{|l|}{$\begin{array}{l}\text { Características } \\
\text { sociodemográficas }\end{array}$} \\
\hline Sexo $(1=$ hombre $)$ & & .084 & . & & & & & . & & .073 & \\
\hline Edad & . & & .094 & .074 & . & . & .091 & & & .092 & . \\
\hline Escolaridad & .127 & .127 & .175 & .127 & .183 & .081 & .205 & .128 & .152 & .165 & . \\
\hline $\begin{array}{l}\text { Autopercepción de ingresos } \\
\text { razonables }\end{array}$ & . & & .074 & . & .147 & . & .093 & & . & . & . \\
\hline NSE & . & & .139 & . & -.127 & . & . & . & . & . & . \\
\hline \multicolumn{12}{|l|}{ Confianza interpersonal } \\
\hline $\begin{array}{l}\text { Se puede confiar en la mayo- } \\
\text { ría de las personas }(1=\text { sí) }\end{array}$ & .107 & . & . & . & -.087 & & . & . & . & & -.260 \\
\hline \multicolumn{12}{|l|}{ Exposición a los medios (TV) } \\
\hline $\begin{array}{l}N^{\circ} \text { de días a la semana que } \\
\text { ve las noticias en TV }\end{array}$ & & .072 & .092 & & .093 & & & . & & .092 & \\
\hline \multicolumn{12}{|l|}{$\begin{array}{l}\text { Evaluación de la situación } \\
\text { económica }\end{array}$} \\
\hline $\begin{array}{l}\text { Evaluación de la situación } \\
\text { económica personal }\end{array}$ & .068 & & & . & -.132 & .096 & . & & . & . & . \\
\hline $\begin{array}{l}\text { Evaluación de la situación } \\
\text { económica del país }\end{array}$ & & & . & & & & & . & & .111 & \\
\hline \multicolumn{12}{|l|}{$\begin{array}{l}\text { Evaluación de la situación } \\
\text { política }\end{array}$} \\
\hline $\begin{array}{l}\text { Aprobación de la gestión del } \\
\text { presidente }(1=\text { sí) }\end{array}$ & . & & & & -.205 & .246 & .068 & .137 & -.069 & .151 & \\
\hline $\begin{array}{l}\text { Calificación de la situación } \\
\text { política del país }\end{array}$ & . & & .094 & & . & . & & . & & . & . \\
\hline \multicolumn{12}{|l|}{$\begin{array}{l}\text { Satisfacción con la } \\
\text { democracia }\end{array}$} \\
\hline $\begin{array}{l}\text { Satisfacción con el funciona- } \\
\text { miento de la democracia }\end{array}$ & .283 & .251 & .194 & .250 & .217 & . & .167 & .121 & .255 & .193 & -.089 \\
\hline R Cuadrado corregida & .128 & .123 & .167 & .099 & .127 & .115 & .106 & .050 & .156 & .157 & .071 \\
\hline
\end{tabular}

15 La evidencia empírica a "nivel de análisis agregado" tampoco permite ratificar dicho supuesto. En un estudio en el que se incluyen 129 países, Solimano (2010: 61-63) encuentra que la correlación entre el tamaño relativo de la clase media y la democracia (medida a través del índice Polity IV) es más bien baja o nula para la mayoría de los países, excepto para las economías de más altos ingresos. 
Tabla 5. Determinantes del Índice de Apoyo a la Democracia en países con ingreso per cápita medio bajo, 2011

\begin{tabular}{|c|c|c|c|c|c|c|}
\hline & Bolivia & El Salvador & Guatemala & Honduras & Nicaragua & Paraguay \\
\hline \multicolumn{7}{|l|}{ Características sociodemográficas } \\
\hline Sexo (1 = hombre) & .065 & . & . & . & .086 & \\
\hline Edad & & & . & . & . & \\
\hline Escolaridad & . & . & . & . & .111 & .131 \\
\hline Autopercepción de ingresos razonables & . & . & . & . & . & . \\
\hline NSE & . & . & . & . & . & . \\
\hline \multicolumn{7}{|l|}{ Confianza interpersonal } \\
\hline $\begin{array}{l}\text { Se puede confiar en la mayoría } \\
\text { de las personas }(1=\mathrm{si})\end{array}$ & . & -.077 & . & . & -.076 & -.073 \\
\hline \multicolumn{7}{|l|}{ Exposición a los medios (TV) } \\
\hline $\begin{array}{l}N^{\circ} \text { de días a la semana que ve } \\
\text { las noticias en TV }\end{array}$ & .113 & .076 & .122 & . & . & . \\
\hline \multicolumn{7}{|l|}{ Evaluación de la situación económica } \\
\hline $\begin{array}{l}\text { Evaluación de la situación económica } \\
\text { personal }\end{array}$ & . & . & . & & . & . \\
\hline $\begin{array}{l}\text { Evaluación de la situación económica } \\
\text { del país }\end{array}$ & . & . & . & -.112 & . & .078 \\
\hline \multicolumn{7}{|l|}{ Evaluación de la situación política } \\
\hline $\begin{array}{l}\text { Aprobación de la gestión del presidente } \\
(1=s i ́)\end{array}$ & & . & . & . & . & .107 \\
\hline $\begin{array}{l}\text { Calificación de la situación política } \\
\text { del país }\end{array}$ & . & . & . & . & . & . \\
\hline \multicolumn{7}{|l|}{ Satisfacción con la democracia } \\
\hline $\begin{array}{l}\text { Satisfacción con el funcionamiento } \\
\text { de la democracia }\end{array}$ & .235 & .297 & .261 & .174 & .205 & .295 \\
\hline R Cuadrado corregida & .076 & .147 & .104 & .047 & .059 & .179 \\
\hline
\end{tabular}

\section{Conclusiones}

En primer lugar, cabe señalar que, luego de más de dos décadas de que en América Latina se retornó a la democracia, los niveles de adhesión a esta — como la mejor forma de gobierno- son todavía bajos. Nuestras democracias no han alcanzado niveles de legitimidad satisfactorios comparables con los de las democracias desarrolladas, situación que vuelve a la cultura cívico-democrática de los latinoamericanos especialmente débil. Durante el periodo 1996-2011, el grado de apoyo a la democracia ha sido particularmente bajo en algunos países. En 2011, solo seis de los diecisiete países estudiados presentan niveles de apoyo a la democracia por sobre el $66 \%$, cifra que es significativamente menor si se la compara con el Índice de Apoyo a la Democracia aquí elaborado. Esto es algo que debiera preocuparnos, en la medida en que existe un amplio consenso en que la estabilidad y la calidad de la democracia dependen del grado de apoyo que le brinde la ciudadanía. Tal y como ha señalado el Programa de las Naciones Unidas para el Desarrollo, en su informe La democracia en América 
Latina: Hacia una democracia de ciudadanas y ciudadanos, "el apoyo que dan los ciudadanos a la democracia es un elemento clave de su sustentabilidad [...] Las democracias se tornan vulnerables cuando, entre otros factores, las fuerzas políticas autoritarias encuentran en las actitudes ciudadanas terreno fértil para actuar" (PNUD, 2004: 137). En otras palabras, "[si] las mayorías dejan de apoyar al sistema democrático, se desvanece la legitimidad y, a la larga, se pierde la sostenibilidad" (PNUD, 2010: 37).

En segundo lugar, al examinar la relación entre el NSE y el apoyo a la democracia en la región a nivel agregado durante el periodo 1996-2011, se constata que, a medida que aumenta el NSE, tiende a aumentar también la proporción de quienes consideran la democracia como la mejor forma de gobierno. Es decir, los segmentos medios presentan, en promedio, mayores niveles de apoyo a la democracia que el NSE bajo, y menores que los que se observan en el NSE alto. Pero al examinar dicha relación por países, se constata que esta tiende a desaparecer en la mayoría de los casos durante el periodo 1996-2011, con la excepción de Argentina, Brasil, Chile, Colombia, México y Perú. En particular, la hipótesis de Lipset (la clase más baja en cualquier país tiende a ser más autoritaria que las clases superiores) solamente se verifica en ocho de los diecisiete países estudiados en 2011 (principalmente en aquellos con ingreso per cápita medio alto). De igual modo, el desarrollo económico experimentado por los países latinoamericanos no ha implicado necesariamente la emergencia o consolidación de una clase media que manifieste "claramente" mayores niveles de apoyo a la democracia que el resto de los segmentos sociales, especialmente respecto del NSE alto.

En tercer lugar, al retestear los resultados anteriores a partir de la técnica de regresión lineal múltiple, sobre la base de la inclusión de un conjunto de variables de control y la elaboración de un Índice de Apoyo a la Democracia, es posible concluir con mayor precisión que solo en dos de los diecisiete países analizados en 2011 se verifica la relación entre NSE y apoyo a la democracia (ambos con un nivel de desarrollo económico medio alto). Y mientras que, en el caso de Colombia se da una relación directamente proporcional, en el caso de Chile esta se da en el sentido contrario. Tales resultados ponen en entredicho la pertinencia de las hipótesis derivadas de las teorías de la modernización y las de la posmodernización para la gran mayoría de los países durante el periodo estudiado. Por otra parte, la evidencia a favor del enfoque contingente es igualmente débil. Ambas aproximaciones resultan inapropiadas para los países latinoamericanos, ya que se inclinan a sobreestimar las diferencias y el impacto que tiene el NSE en las actitudes hacia la democracia. ${ }^{16}$

16 Existe una visión pesimista acerca de la propensión de las personas pobres a apoyar y formar parte de la democracia (Krishna, 2008: 2-3, 6), cuestión que, aunque plausible, no cuenta con suficiente eviden- 
En tal sentido, en cuarto lugar, las teorías de la democracia que han enfatizado la estructura de clases constituyen explicaciones inapropiadas para comprender la transformación que ha experimentado la región a partir de 1978 (Mainwaring, y Pérez-Liñán, 2005: 14). Así, por ejemplo, en las últimas décadas, los países latinoamericanos han logrado un importante incremento en los niveles educativos de su población (Fukuyama, 2009: 150), incluidos los segmentos más pobres. Esto podría explicar, al menos en parte, el hecho de que no exista una incidencia estadísticamente significativa del NSE en el Índice de Apoyo a la Democracia en la mayoría de los países examinados. Los datos sugieren que entre las características sociodemográficas, más que el NSE de las personas, es la escolaridad lo que determina en mayor medida los niveles de apoyo a la democracia. En otras palabras, una mayor escolaridad conllevaría actitudes más democráticas. Y a pesar de que el mecanismo mediante el cual esto ocurre todavía no es bien comprendido (Lipset, y Lakin, 2004: 168), es muy probable que conforme aumenta la escolaridad, las personas amplíen su conocimiento y comprensión acerca del valor e importancia de un sistema democrático (Krishna, 2008: 26). No obstante lo anterior, la variable que más incide sobre el Índice de Apoyo a la Democracia es la "satisfacción con el funcionamiento de la democracia”.

Finalmente, si bien hay acuerdo general en que el proceso de democratización es más estable cuando tanto los líderes políticos como los ciudadanos comparten y apoyan una cierta visión de la democracia (Dalh, 2000: 157, 181; Sarsfield, 2010: 18-9, 21; Diamond, 2008: 154-157; Mainwaring, y Hagopian, 2005: 8), siendo las orientaciones de los ciudadanos claves para comprender el proceso de democratización de nuestras sociedades (Welzel, y Inglehart, 2008: $26,28-29)$, es igualmente relevante realizar estudios que incorporen tanto las explicaciones de los agentes como las de carácter estructural, prestando especial atención a la interacción entre ambos niveles (Elgströn, 2002: 198).

\section{Referencias}

Banco Mundial, 2011, The World Development Report 2012: Gender Equality and Development, Washington, DC, The World Bank Press.

Booth, John, y Mitchell Seligson, 2009, The Legitimacy Puzzle in Latin America. Political Support and Democracy in Eight Nations, Cambridge, Cambridge University Press.

cia empírica desde la perspectiva comparada en los países en vías de desarrollo, particularmente en un nivel de análisis individual (Krishna, 2008: 6-8). 
Cardoso, Fernando H., y Enzo Faletto, 1969, Dependencia y desarrollo en América Latina, Buenos Aires, Siglo XxI.

Chen, Jie, 2013, A Middle Class without Democracy: Economic Growth and the Prospects for Democratization in China, Oxford, Oxford University Press.

Chu, Yun-han et al., 2008, "Public Opinion and Democratic Legitimacy", en Larry Diamond y Marc F. Plattner (eds.), How People View Democracy, Baltimore, MD, The Johns Hopkins University Press, pp. 31-44.

Dahl, Robert, 2000, On Democracy, New Haven, Yale University Press.

Dalton, Russell J., Doch C. Shin, y Willy Jou, 2008, “How People Understand Democracy”, en Larry Diamond, y Marc F. Plattner (eds.), How People View Democracy, Baltimore, MD, The Johns Hopkins University Press, pp. 1-15.

Dalton, Russell J., 2004, Democratic Challenges. Democratic Choices: The Erosion of Political Support in Advanced Industrial Democracies, Oxford, Oxford University Press.

Dalton, Russell J., 1999, "Political Support in Advanced Industrial Democracies", en Pippa Norris (ed.), Critical Citizens. Global Support for Democratic Governance, Oxford, Oxford University Press, pp. 57-77.

Diamond, Larry, 2008, The Spirit of Democracy. The Struggle to Build Free Societies throughout the World, Nueva York, Times Books.

Diamond, Larry, 1999, Developing Democracy. Towards Consolidation, Baltimore y Londres, The Johns Hopkins University Press.

Doorenspleet, Renske, 2002, "Development, Class and Democracy: is There a Relationship?, en Ole Elgström, y Goran Hyden, Development and Democracy. What Have We Learned and How?, Londres y Nueva York, Routledge, pp. 48-64.

Easton, David, 1975, "A Re-assessment of the Concept of Political Support", British Journal of Political Science, vol. 5, núm. 4, pp. 435-457.

Elgström, Ole, 2002, "Development and Democracy: Gains and Gaps?, en Ole Elgström y Goran Hyden, Development and Democracy. What Have We Learned and How?, Londres y Nueva York, Routledge, pp. 191-201.

Ferreira, Francisco H. G., Julian Messina, Jamele Rigolini, Luis-Felipe López-Calva, María Ana Lugo, y Renos Vakis, 2013, Economic Mobility and the Rise of the Latin American Middle Class, Washington, The World Bank. 
Franco, Rolando et al., 2011, "Crece y cambia la clase media en América Latina: una puesta al día”, Revista CEPAL, núm. 103, pp. 7-26.

Franco, Rolando, y Martín Hopenhayn, 2010, "Las clases medias en América Latina: Historias cruzadas y miradas diversas”, en Rolando Franco, Martín Hopenhayn, y Arturo León (coords.), Las clases medias en América Latina. Retrospectiva y nuevas tendencias, México, CEPAL/Siglo XXI, pp. 7-41.

Franco, Rolando, y Arturo León, 2010, “Clases medias latinoamericanas: ayer y hoy”, Estudios Avanzados, núm. 13, pp. 59-77.

Fukuyama, Francis, 2009, "La experiencia latinoamericana”, Journal of Democracy en Español, núm. 1, pp. 147-159.

Furtado, Celso, 1964, Desarrollo y subdesarrollo, Buenos Aires, Eudeba.

Gunther, Richard et al., 2007a, "Introduction: Intermediation, Information, and Electoral Politics", en Richard Gunther et al., Democracy, Intermediation and Voting in Four Continents, Oxford, Oxford University Press, pp. 1-28.

Gunther, Richard et al., 2007b, "Conclusions: Processes of Intermediation, Electoral Politics, and Political Support in Old a New Democracies", en Richard Gunther et al., Democracy, Intermediation and Voting in Four Continents, Oxford, Oxford University Press, pp. 321-367.

Graham, Carol, y Sandip Sukhtankar, 2004, "Does Economic Crisis Reduce Support for Markets and Democracy in Latin America? Some Evidence from Surveys of Public Opinion and Well Being", Journal of Latin American Studies, vol. 36, núm. 2, pp. 449-377.

Hopenhayn, Martín, 2010a, “¿Cómo ha cambiado la clase media en América Latina? Elementos para el debate”, en Ludolfo Paramio (coord.), Clases medias y gobernabilidad en América Latina, Madrid, Editorial Pablo Iglesias, pp. 25-57.

Hopenhayn, Martín, 2010b, "Clases medias en América Latina: sujeto difuso en busca de definición”, en Alicia Bárcena, y Narcís Serra (eds.), Clases medias y desarrollo en América Latina, Santiago de Chile, CEPAL/CIDOB, pp. 11-37.

Huntington, Samuel P., 1993, The Third Wave: Democratization in the Late Twentieth Century, Norman, University of Oklahoma Press.

Inglehart, Ronald, 2000, Modernización y posmodernización. El cambio cultural, económico y politico en 43 sociedades, Madrid, cis. 
Inglehart, Ronald, 1977, The Silent Revolution: Changing Values and Political Styles among Western Publics, Princeton, Princeton University Press.

Inglehart, Ronald, y Christian Welzel, 2006, Modernización, cambio cultural y democracia: La secuencia del desarrollo humano, Madrid, cis.

Klingemann, Hans-Dieter, 1999, "Mapping Political Support in the 1990s: A Global Analysis", en Pippa Norris (ed.), Critical Citizens. Global Support for Democratic Governance, Oxford, Oxford University Press, pp. 31-56.

Krishna, Anirudh, 2008, "1. Introduction: Poor People and Democracy”, en Anirudh Krishna (ed.), Poverty, Participation and Democracy. A global Perspective, Cambridge, Cambridge University Press, pp. 1-27.

Kurlantzick, Joshua, 2013, Democracy in Retreat. The Revolt of the Middle Class and the Worldwide Decline of Representative Government, New Haven y Londres, Yale University Press.

Latinobarómetro, 2006, Informe Latinobarómetro 2006, Santiago de Chile, Latinobarómetro.

León, Arturo et al., 2010, "Clases medias en América Latina: Una visión de sus cambios en las dos últimas décadas”, en Rolando Franco, Martín Hopenhayn, y Arturo León (coords.), Las clases medias en América Latina: Retrospectiva y nuevas tendencias, México, CEPAL/Siglo XXI, pp. 43-116.

Lipset, Seymour Martin, 1960, Political Man. The Social Bases of Politics, Nueva York, Doubleday \& Company.

Lipset, Seymour Martin, 1959a, "Some Social Requisites of Democracy: Economic Development and Political Legitimacy", The American Political Review, vol. 53, núm. 1, pp. 69-105.

Lipset, Seymour Martin, 1959b, "Democracy and Working-Class Authoritarianism”, American Sociological Review, vol. 24, núm. 4, pp. 482-501.

Lipset, Seymour Martin, y Jason M. Lakin, 2004, The Democratic Century, Oklahoma, University of Oklahoma Press.

López-Calva, Luis F., Jamele Rigolini, y Flerencia Torche, 2011, "Is There Such a Thing as Middle Class Values? Class Differences, Values and Political Orientations in Latin America", Policy Research Working Paper, núm. 5874.

Mainwaring, Scott, y Frances Hagopian, 2005, "Introduction: The Third Wave of Democratization in Latin America, en Frances Hagopian, y Scott Mainwaring, The Third Wave of De- 
mocratization in Latin America. Advances and Setbacks, Cambridge, Cambridge University Press, pp. 1-13.

Mainwaring, Scott, y Aníbal Pérez-Liñán, 2005, "Latin American Democratization since 1978. Democratic Transitions, Breakdowns, and Erosions”, en Frances Hagopian, y Scott Mainwaring, The Third Wave of Democratization in Latin America. Advances and Setbacks, Cambridge, Cambridge University Press, pp. 14-59.

Martinelli, Alberto, 2005, Global Modernization. Rethinking the Project of Modernity, Londres, Sage Publications.

Minujin, Alberto, 2010, "Vulnerabilidad y resiliencia de la clase media en América Latina", en Alicia Bárcena, y Narcís Serra (eds.), Clases medias y desarrollo en América Latina, Santiago de Chile, CEPAL/Cidob, pp. 71-141.

Mora y Araujo, Manuel, 2010, "Vulnerabilidad de las clases medias en América Latina. Competitividad individual y posición social”, en Alicia Bárcena, y Narcís Serra (eds.), Clases medias y desarrollo en América Latina, Santiago de Chile, CEPAL/CIDOB, pp. 143-172.

Norris, Pippa, 2011, Democratic Deficit. Critical Citizens Revisited, Cambridge, Cambridge University Press.

Norris, Pippa, 1999, "Introduction: The Growth of Critical Citizens?”, en Pippa Norris (ed.), Critical Citizens. Global Support for Democratic Governance, Oxford, Oxford University Press, pp. 1-27.

oCDE, 2010, Perspectivas económicas de América Latina 2011. En qué medida es clase media América Latina, París, OCDE Publishing.

Paramio, Ludolfo, 2010a, "Economía y política de las clases medias en América Latina", Nueva Sociedad, núm. 229, pp. 62-75.

Paramio, Ludolfo, 2010b, "Introducción: clases medias y gobernabilidad en América Latina", en Ludolfo Paramio (coord.), Clases medias y gobernabilidad en América Latina, Madrid, Editorial Pablo Iglesias, pp. 7-23.

PNUD, 2010, Nuestra democracia, México, PNUD.

PNUD, 2004, La democracia en América Latina: Hacia una democracia de ciudadanas y ciudadanos, Nueva York, PNUD.

Ravallion, Martin, 2010, The Developing World's Bulging (but Vulnerable) "Middle Class", World Development, vol. 38, núm. 4, pp. 445-454. 
Sarsfield, Rodolfo, 2010, "Introducción: democracia, opinión pública y democratización”, en Rodolfo Sarsfield (coord.), Democracia y opinión pública en países de reciente democratización, Madrid, Fundación Pablo Iglesias, pp. 5-50.

Schmitt-Beck, Rüdiger, y Katrin Voltmer, 2007, "The Mass Media in Third-wave Democracies: Gravediggers or Seedsmen of Democratic Consolidation?”, en Richard Gunther et al., Democracy, Intermediation and Voting in Four Continents, Oxford, Oxford University Press, pp. 75-134.

Solimano, Andrés, 2010, "La clase media y el proceso de desarrollo económico: evidencia internacional de 130 países”, en Alicia Bárcena, y Narcís Serra (eds.), Clases medias y desarrollo en América Latina, Santiago de Chile, CEPAL/Cidob, pp. 39-70.

Smith, Peter H., 2005, Democracy in Latin America: Political Change in Comparative Perspective, Nueva York y Oxford, Oxford University Press.

Sztompka, Pior, 1995, “Capítulo 9. Teorías viejas y nuevas de la modernización”, en Sociología del cambio social, Madrid, Alianza, pp. 155-167.

Tavares de Almeida, María Herminia, y Emmanoel Nunes de Oliveira, 2010, "Nuevas capas medias y política en Brasil", en Ludolfo Paramio (coord.), Clases medias y gobernabilidad en América Latina, Madrid, Editorial Pablo Iglesias, pp. 103-118.

Tedesco, Laura, y Jonathan Barton, 2004, The State of Democracy in Latin America. Post-transitional conflicts in Argentina and Chile, Nueva York, Routledge.

Welzel, Christian, y Ronald Inglehart, 2008, "The Role of Ordinary People in Democratization”, en Larry Diamond y Marc F. Plattner (eds.), How People View Democracy, Baltimore, MD, The Johns Hopkins University Press, pp. 16-30.

Welzel, Christian, y Ronald Inglehart, 2007, "Mass Beliefs and Democratic Institutions", en Carles Boix y Susan C. Stokes (eds.), The Oxford Handbook of Comparative Politics, Oxford, Oxford University Press, pp. 297-316.

Recibido el 26 de septiembre de 2013. Aceptado el 27 de octubre de 2014. 